\title{
Envelope ADI-FDTD Method and Its Application in Three-Dimensional Nonuniform Meshes
}

\author{
Shu-Hai Sun and Charles T. M. Choi, Senior Member, IEEE
}

\begin{abstract}
The envelope alternating-direction-implicit finite difference time domain (ADI-FDTD) method in 3-D nonuniform meshes was proposed and studied. The phase velocity error for the envelope ADI-FDTD and ADI-FDTD methods in uniform and nonuniform meshes and different temporal increments were studied. A cavity problem was studied using the envelope ADI-FDTD and ADI-FDTD methods in graded meshes and the conventional FDTD method in a uniform mesh. The simulation results show that the envelope ADI-FDTD performs better than the ADI-FDTD in numerical accuracy.
\end{abstract}

Index Terms-Courant-Friedrich-Levy (CFL) stability condition, envelope alternating-direction-implicit finite difference time domain (ADI-FDTD) method, phase velocity.

\section{INTRODUCTION}

$\mathbf{T}$ THE idea of coupling the wave-envelope technique [1] with the ADI-FDTD method was proposed [2]-[4]. The numerical characteristic of the 3-D complex envelope ADI-FDTD method was derived in analytic form [5] and studied by solving a cavity problem [6]. However, the performance and efficiency of the envelope ADI-FDTD method with nonuniform mesh distribution has not been studied. In this letter, the numerical accuracy of the 3-D envelope ADI-FDTD method with uniform and nonuniform meshes were studied, and the simulation results of a cavity problem are shown.

\section{METHOD}

With the envelope ADI-FDTD method, the wave envelopes of the electromagnetic-field components are modeled, as opposed to the ADI-FDTD method, which simulates the actual electromagnetic-field components. Applying the wave envelope technique [1], the electromagnetic-field components are defined as

$\vec{E}_{(x, y, z, t)}=\vec{E}_{a(x, y, z, t)} \cdot e^{j \omega_{c} t}, \quad \vec{H}_{(x, y, z, t)}=\vec{H}_{a(x, y, z, t)} \cdot e^{j \omega_{c} t}$

where $\vec{E}_{a}$ and $\vec{H}_{a}$ are the wave envelopes and $\omega_{c}$ is the carrier angular frequency. Substituting (1) into Maxwell's curl equations and expanding in Cartesian coordinate into twelve differential equations. Following the FDTD method and ADI technique, the process of updating the electromagnetic-field components in a

Manuscript received August 8, 2006; revised December 7, 2006. This work was supported by the National Science Council of Taiwan, R.O.C., under Grant 95-2221-E-009-365-MY2.

S.-H. Sun is with the Department of Electrical Engineering, I-Shou University, Kaohsiung 840, Taiwan, R.O.C.

C. T. M. Choi is with the Department of Computer Engineering, National Chiao Tung University, Hsinchu 300, Taiwan, R.O.C. (e-mail: c.t.choi@ieee. org).

Digital Object Identifier 10.1109/LMWC.2007.892937 time step in the FDTD method are divided into two half-time steps. These updating equations at each time step are shown as

$$
\begin{aligned}
& \varepsilon_{0}\left(D_{t}+j \omega_{c}\right) E_{x a y}^{N_{1}}+\sigma_{y} E_{x a y}^{N_{1}}=D_{y}\left(H_{z a x}^{N_{2}}+H_{z z a y}^{N_{2}}\right) \\
& \varepsilon_{0}\left(D_{t}+j \omega_{c}\right) E_{x a z}^{N_{1}}+\sigma_{z} E_{x a z}^{N_{1}}=-D_{z}\left(H_{y a x}^{N_{3}}+H_{y a z}^{N_{3}}\right) \\
& \varepsilon_{0}\left(D_{t}+j \omega_{c}\right) E_{y a z}^{N_{1}}+\sigma_{z} E_{y a z}^{N_{1}}=D_{z}\left(H_{x a y}^{N_{2}}+H_{x a z}^{N_{2}}\right) \\
& \varepsilon_{0}\left(D_{t}+j \omega_{c}\right) E_{y a x}^{N_{1}}+\sigma_{x} E_{y a x}^{N_{1}}=-D_{x}\left(H_{z a x}^{N_{3}}+H_{z a y}^{N_{3}}\right) \\
& \varepsilon_{0}\left(D_{t}+j \omega_{c}\right) E_{z a x}^{N_{1}}+\sigma_{x} E_{z a x}^{N_{1}}=D_{x}\left(H_{y a x}^{N_{2}}+H_{y a z}^{N_{2}}\right) \\
& \varepsilon_{0}\left(D_{t}+j \omega_{c}\right) E_{z a y}^{N_{1}}+\sigma_{y} E_{z a y}^{N_{1}}=-D_{y}\left(H_{x a y}^{N_{3}}+H_{x a z}^{N_{3}}\right) \\
& \mu_{0}\left(D_{t}+j \omega_{c}\right) H_{x a y}^{N_{1}}+\sigma_{y} \eta_{0}^{2} H_{x a y}^{N_{1}}=-D_{y}\left(E_{z a x}^{N_{3}}+E_{z a y}^{N_{3}}\right) \\
& \mu_{0}\left(D_{t}+j \omega_{c}\right) H_{x a z}^{N_{1}}+\sigma_{z} \eta_{0}^{2} H_{x a z}^{N_{1}}=D_{z}\left(E_{y a x}^{N_{2}}+E_{y a z}^{N_{2}}\right) \\
& \mu_{0}\left(D_{t}+j \omega_{c}\right) H_{y a z}^{N_{1}}+\sigma_{z} \eta_{0}^{2} H_{y a z}^{N_{1}}=-D_{z}\left(E_{x a y}^{N_{3}}+E_{x a z}^{N_{3}}\right) \\
& \mu_{0}\left(D_{t}+j \omega_{c}\right) H_{y a x}^{N_{1}}+\sigma_{x} \eta_{0}^{2} H_{y a x}^{N_{1}}=D_{x}\left(E_{z a x}^{N_{2}}+E_{z a y}^{N_{2}}\right) \\
& \mu_{0}\left(D_{t}+j \omega_{c}\right) H_{z a x}^{N_{1}}+\sigma_{x} \eta_{0}^{2} H_{z a x}^{N_{1}}=-D_{x}\left(E_{y a x}^{N_{3}}+E_{y a z}^{N_{3}}\right) \\
& \mu_{0}\left(D_{t}+j \omega_{c}\right) H_{z a y}^{N_{1}}+\sigma_{y} \eta_{0}^{2} H_{z a y}^{N_{1}}=D_{y}\left(E_{x a y}^{N_{2}}+E_{x a z}^{N_{2}}\right)
\end{aligned}
$$

where $D_{x}, D_{y}, D_{z}$, and $D_{t}$ represent the first-order central finite-difference operators in $x, y, z$ directions, and time domain, respectively; $\eta_{0}$ is the free-space wave impedance, $\sigma_{s}$ is electric conductivity, and $s=x, y$, or $z . E_{x a y}^{N_{1}}$ in (2a) means the $E_{x a y}$ component locates at the $N_{1}$ time step. From the $n$ time step to the $n+1$ time step, $N_{1}, N_{2}, N_{3}$ in the first half-time step are $n+1 / 4, n+1 / 2, n$, respectively; $N_{1}, N_{2}, N_{3}$ in the second half-time step are $n+3 / 4, n+1 / 2, n+1$, respectively. The electromagnetic-field components are distributed in graded meshes as shown in Fig. 1(a), and the discrete forms of (2e) and (2j) in the first half-time step are shown below as examples:

$$
\begin{aligned}
& E_{z a x,(i, j, k+1 / 2)}^{n+1 / 2} \\
& =c a_{x,(i, j, k+1 / 2)} E_{z a x,(i, j, k+1 / 2)}^{n}+c b_{x,(i, j, k+1 / 2)} \\
& \times\left[H_{\text {yax },(i+1 / 2, j, k+1 / 2)}^{n+1 / 2}+H_{y a z,(i+1 / 2, j, k+1 / 2)}^{n+1 / 2}\right. \\
& \left.-H_{y a x,(i-1 / 2, j, k+1 / 2)}^{n+1 / 2}-H_{y a z,(i-1 / 2, j, k+1 / 2)}^{n+1 / 2}\right] \\
& H_{\text {yax },(i+1 / 2, j, k+1 / 2)}^{n+1 / 2} \\
& =d a_{x,(i+1 / 2, j, k+1 / 2)} H_{y a x,(i+1 / 2, j, k+1 / 2)}^{n} \\
& +d b_{x,(i+1 / 2, j, k+1 / 2)^{\circ}} \\
& \times\left[E_{z a x,(i+1, j, k+1 / 2)}^{n+1 / 2}+E_{z a y,(i+1, j, k+1 / 2)}^{n+1 / 2}\right. \\
& \left.-E_{z a x,(i, j, k+1 / 2)}^{n+1 / 2}-E_{z a y,(i, j, k+1 / 2)}^{n+1 / 2}\right]
\end{aligned}
$$

where the coefficients are written as

$$
\begin{aligned}
& c a_{s,(i, j, k)} \\
& =\frac{4 \varepsilon_{0}-\sigma_{s,(i, j, k)} \Delta t-j \varepsilon_{0} \omega_{c} \Delta t}{4 \varepsilon_{0}+\sigma_{s,(i, j, k)} \Delta t+j \varepsilon_{0} \omega_{c} \Delta t}
\end{aligned}
$$




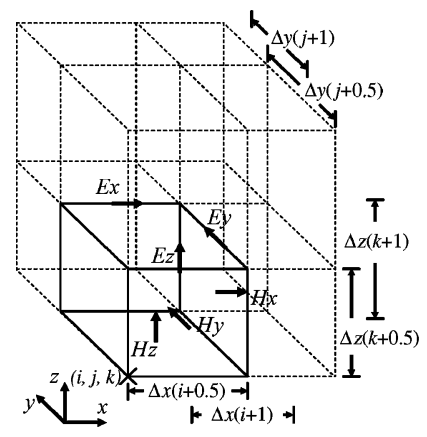

(a)

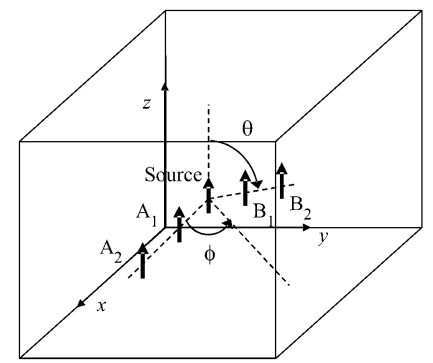

(b)
Fig. 1. In a 3-D domain: (a) a graded mesh and (b) a 3-D model used for studying the phase velocity error.

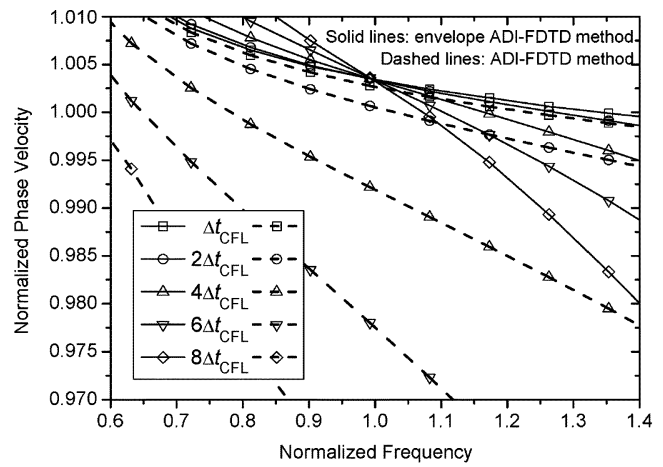

(a)

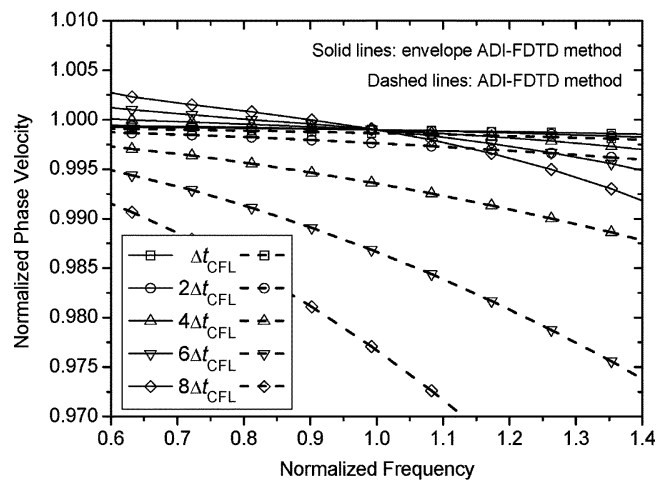

(b)

Fig. 2. Normalized phase velocity versus the normalized frequency. The cell size is $c_{0} /\left(40 f_{c}\right)$. The propagation direction is (a) $A_{1}$ to $A_{2}$ and (b) $B_{1}$ to $B_{2}$.

$$
\begin{aligned}
& c b_{s,(i, j, k)} \\
& =2 \Delta t \\
& \quad \cdot\left[\Delta s_{(i, j, k)}\left(4 \varepsilon_{0}+\sigma_{s,(i, j, k)} \Delta t+j \varepsilon_{0} \omega_{c} \Delta t\right)\right]^{-1} \\
& d a_{s,(i, j, k)} \\
& =\frac{4 \mu_{0}-\sigma_{s,(i, j, k)} \eta_{0}^{2} \Delta t-j \mu_{0} \omega_{c} \Delta t}{4 \mu_{0}+\sigma_{s,(i, j, k)} \eta_{0}^{2} \Delta t+j \mu_{0} \omega_{c} \Delta t} \\
& d b_{s,(i, j, k)} \\
& =2 \Delta t \\
& \quad \cdot\left[\Delta s_{(i, j, k)}\left(4 \mu_{0}+\sigma_{s,(i, j, k)} \eta_{0}^{2} \Delta t+j \mu_{0} \omega_{c} \Delta t\right)\right]^{-1} .
\end{aligned}
$$

In these half-time steps, the implicit formulations can be solved with a tridiagonal matrix [7]. At the conclusion of the simulation, the wave envelope is modulated by the carrier to obtain the actual wave propagation.

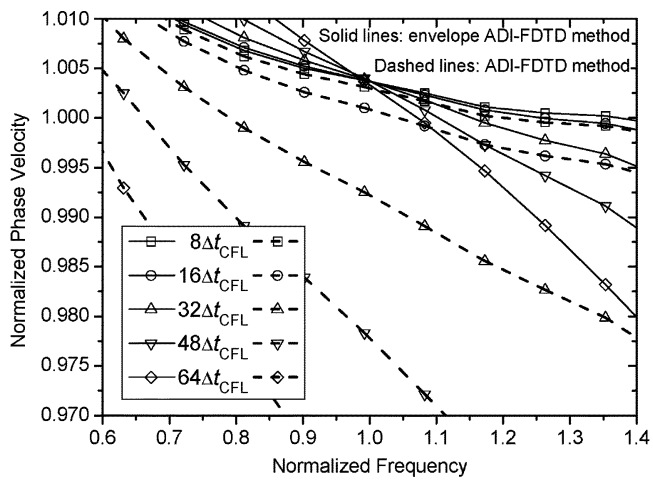

(a)

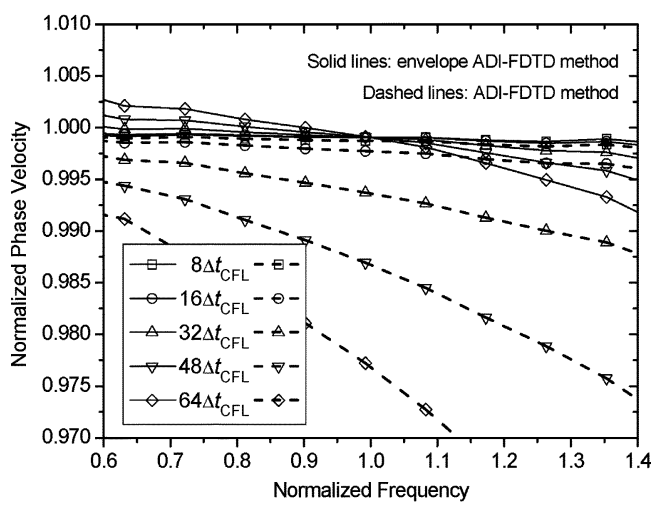

(b)

Fig. 3. Normalized phase velocity versus the normalized frequency. The maximum cell size is $c_{0} /\left(40 f_{c}\right)$. The propagation direction is (a) $A_{1}$ to $A_{2}$ and (b) $B_{1}$ to $B_{2}$

\section{RESULTS}

\section{A. Study of the Numerical Phase Velocity}

The numerical phase velocity was studied by simulating the wave propagation in the free-space model as shown in Fig. 1(b). The dimension of the free space is $300 \times 300 \times 300$ cells. In order to obtain more accurate simulation results, 20 layers of the PML medium were imposed outside. A Gaussian pulse with bandwidth $f_{e}$ of $6.35 \mathrm{GHz}$ modulated with a carrier frequency $f_{c}$ of $15 \mathrm{GHz}$ was introduced at the source point which is located at $(151,151,151)$ in free space. Points $A_{1}$ and $A_{2}$, which are located at $(231,151,151)$ and $(251,151,151)$, respectively, are used to study the wave propagation along the $x$-axis $\left(\theta=90^{\circ}\right.$ and $\phi=0^{\circ}$ ). Points $B_{1}$ and $B_{2}$, which are located at (231, $231,231)$ and $(251,251,251)$, respectively, are used to model the oblique wave propagation in $x y z$ domain $\left(\theta=45^{\circ}\right.$ and $\phi=45^{\circ}$ ). At the end of the simulation, the numerical phase velocity can be computed [7].

In the following discussion, the free space is discretized in an uniform mesh (model 1) and a nonuniform mesh (model $2)$. In model 1 , the cell size $\Delta$ is $c_{0} /\left(40 f_{c}\right)$, and the maximum temporal increment under the CFL stability condition is $\Delta t_{\mathrm{CFL}}=\Delta /\left(\sqrt{3} \mathrm{c}_{0}\right)$. The model was simulated for the envelope ADI-FDTD and ADI-FDTD methods with different temporal increments, and the numerical phase velocity normalized by the speed of light versus frequency normalized by the carrier frequency are shown in Fig. 2. Here, the theoretical solution of the normalized phase velocity is 1 . For the same temporal increment, we can find the numerical accuracy of the 


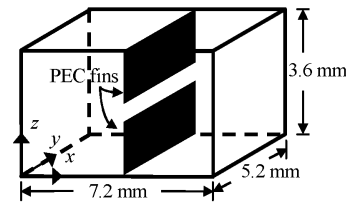

(a)

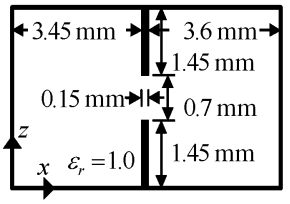

(b)

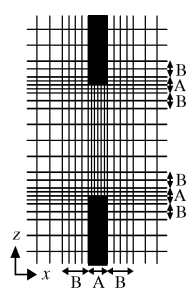

(c)
Fig. 4. A 3-D cavity model. (a) Bird's eye view. (b) Side view. (c) Under side view, the cell distribution near the PEC fins.

TABLE I

RESULTS OF A 3-D CAVITY IN FIG. 4

\begin{tabular}{|c|c|c|c|c|}
\hline \multicolumn{2}{|c|}{$\Delta t / \Delta t_{\mathrm{CFL}}$} & \multicolumn{2}{c|}{ Resonant Frequency $(\mathrm{GHz})$} & \multirow{2}{*}{ CPU time (s) } \\
\cline { 2 - 5 } & 8 & $1^{\text {st }}$ & $2^{\text {nd }}$ & \\
\cline { 2 - 5 } envelope & 16 & 51.096 & 55.296 & 101574.520 \\
\cline { 2 - 5 } ADI-FDTD & 24 & 51.096 & 55.296 & 52153.641 \\
\cline { 2 - 5 } & 32 & 51.139 & 55.296 & 33844.640 \\
\cline { 2 - 5 } & 40 & 51.139 & 55.296 & 25053.016 \\
\cline { 2 - 5 } & 48 & 51.144 & 55.258 & 20099.860 \\
\hline \multirow{5}{*}{ ADI-FDTD } & 8 & 51.052 & 55.166 & 38712.937 \\
\cline { 2 - 5 } & 16 & 50.922 & 54.863 & 19145.515 \\
\cline { 2 - 5 } & 24 & 50.703 & 54.427 & 12980.250 \\
\cline { 2 - 5 } & 32 & 50.359 & 53.867 & 9720.562 \\
\cline { 2 - 5 } & 40 & 50.013 & 53.174 & 7786.750 \\
\cline { 2 - 5 } & 48 & 49.537 & 52.438 & 6483.531 \\
\hline FDTD $^{(1)}$ & 2 & 51.096 & 55.209 & 218253.122 \\
\hline FDTD $^{(2)}$ & 2 & 51.096 & 55.209 & 363966.750 \\
\hline
\end{tabular}

diagonal propagation $\left(\theta=45^{\circ}\right.$ and $\left.\phi=45^{\circ}\right)$ is better than that of the normal propagation $\left(\theta=90^{\circ}\right.$ and $\left.\phi=0^{\circ}\right)$ in Fig. 2, and this result is the same as the theoretical analysis published in [5]. For the envelope ADI-FDTD method, the numerical error for all the temporal increments as shown in Fig. 2 is less than $2 \%$, but the numerical error of the ADI-FDTD method is much larger than $2 \%$ when temporal increment is $8 \Delta t_{\mathrm{CFL}}$ and normalized frequency is 1.0. In model 2 , the distribution of a nonuniform mesh is

$$
\begin{aligned}
& \Delta s(i)=\Delta, \quad 1 \leq i \leq 237, \quad 245 \leq i \leq 300 \\
& \Delta s(i)=\Delta / 2, \quad i=238,244 \\
& \Delta s(i)=\Delta / 2^{2}, \quad i=239,243 \\
& \Delta s(i)=\Delta / 2^{3}, \quad 240 \leq i \leq 242
\end{aligned}
$$

where $s=x, y$, or $z$. Since the maximum cell size is $\Delta \times \Delta \times \Delta$ and the minimum cell size is $\Delta / 8 \times \Delta / 8 \times \Delta / 8$, the $\Delta t_{\mathrm{CFL}}$ here is $1 / 8$ of $\Delta t_{\mathrm{CFL}}$ in model 1 . This model was also modeled with the envelope ADI-FDTD and ADI-FDTD methods, and the simulation results are shown in Fig. 3. We can find each plot in Fig. 3 almost identical to the corresponding plots in Fig. 2, the envelope ADI-FDTD method also maintains good numerical accuracy in 3-D nonuniform mesh distribution.

\section{B. Application of a 3-D Cavity Problem}

The specifications of the structure are shown in Fig. 4. The thicknesses of the two fins are $0.15 \mathrm{~mm}$. A Gaussian pulse with bandwidth $8 \mathrm{GHz}$ modulated with a carrier frequency of $54 \mathrm{GHz}$ was introduced, and this problem was solved by using the ADI-FDTD, the envelope ADI-FDTD, and the traditional FDTD methods. The mesh size in $y$ direction is uniform at $0.1 \mathrm{~mm}$ and in $x, z$ directions are nonuniform. The mesh distribution near the PEC fin is shown in Fig. 4(c), where the definition of the mesh size in $x, z$ directions are $0.025 \mathrm{~mm}$ in segment $\mathrm{A}, 0.050 \mathrm{~mm}$ in segment $\mathrm{B}$, and $0.100 \mathrm{~mm}$ in other mesh. This problem was solved by the ADI-FDTD and the envelope ADI-FDTD methods in nonuniform mesh, and was also solved by the conventional FDTD method in uniform mesh where the cell size was $0.05 \times 0.05 \times 0.05 \mathrm{~mm}^{3}$. Here, $\Delta t_{\mathrm{CFL}}$ is set as $\left(0.025 \times 10^{-3} \mathrm{~m}\right) /\left(c_{0} \sqrt{3}\right)$. The simulation time in the ADI-FDTD and the envelope ADI-FDTD methods is $7.698 \mathrm{~ns}$, and that in the FDTD method is $7.698 \mathrm{~ns}^{(1)}$ and $15.396 \mathrm{~ns}^{(2)}$. We recorded the values of $E_{z}$ field at the observation point, then padded zeros of twice the simulation time were added, and applied the discrete Fourier transform to compute the resonant frequencies of the cavity. The resonant frequencies and CPU time for all three numerical methods are listed in Table I. Since the cavity was discretized by a uniform fine mesh in the FDTD method, we took the results of the FDTD method as the reference. For the first resonant frequency, it is found that the relative error of the envelope ADI-FDTD method is always less than that of the ADI-FDTD method with the same CPU time. The envelope ADI-FDTD method also takes less CPU time and obtains the same value of the first resonant frequency which was solved by the FDTD method. Besides, the numerical error of the ADI-FDTD method increases with the temporal increment, but the envelope ADI-FDTD method always maintains good performance even when the temporal increment is large.

\section{CONCLUSION}

The numerical accuracy and efficiency of the 3-D envelope ADI-FDTD method were presented in this letter. Compared with the ADI-FDTD method, the numerical phase velocity for the envelope ADI-FDTD method is much more accurate than that of the ADI-FDTD method. The numerical solutions of a 3-D cavity problem show that the efficiency of the envelope ADI-FDTD method is better than that of the ADI-FDTD method.

\section{REFERENCES}

[1] C. T. M. Choi and J. P. Webb, "Wave-envelope and transformation methods for finite element solution of unbounded electromagnetic wave problems," IEEE Trans. Magn., vol. 32, no. 3, pp. 886-889, May 1996.

[2] H. Rao, R. Scarmozzino, and R. M. Osgood, "An improved ADI-FDTD method and its application to photonic simulations," IEEE Photon. Technol. Lett., vol. 14, no. 4, pp. 477-479, Apr. 2002.

[3] C. T. M. Choi and S. H. Sun, "Numerical performance and applications of the envelope ADI-FDTD method," IEEE Trans. Microw. Theory Tech., vol. 54, no. 1, pp. 256-264, Jan. 2006.

[4] S. H. Sun and C. T. M. Choi, "Performance of the improved PML for the envelope ADI-FDTD method in two-dimensional domain," IEEE Microw. Wireless Compon. Lett., vol. 15, no. 11, pp. 800-802, Nov. 2005.

[5] C. Ma and Z. Chen, "Dispersion analysis of the three-dimensional complex envelope ADI-FDTD method," IEEE Trans. Microw. Theory Tech., vol. 53, no. 3, pp. 971-976, Mar. 2005.

[6] — "The complex envelope (CE) ADI-FDTD method," in IEEE MTT-S Int. Dig., Jun/ 2005, pp. 1119-1122.

[7] T. Namiki and K. Ito, "Investigation of numerical errors of the twodimensional ADI-FDTD method," IEEE Trans. Microw. Theory Tech., vol. 48 , no. 11 , pp. 1950-1956, Nov. 2000. 